\title{
Immunoexpression of Matrix Metalloproteinase-9 in Histopathological tissue samples of oral Squamous Cell Carcinoma
}

\author{
${ }^{1}$ Senior Lecturer Department of Oral Pathology Women Medical and Dental College Abbottabad. \\ ${ }^{2}$ Assocaite Professor Oral Pathology Department of Oral pathology, Peshawar Medical College \\ ${ }^{3}$ Department of Histopathology Peshawar Medical College \\ ${ }^{4}$ Associate Professor, Head of Department Abbottabad International Medical \& Dental College, Abbottabad. \\ ${ }^{5}$ Associate Professor Department of Oral Biology Lahore Medical \& Dental College \\ ${ }^{6}$ Associate Professor, HOD Department of Oral Biology Women Medical \& Dental College Abbottabad \\ Correspondence to Dr Sikandar Javed Bajwa, Email sikandar.bajwa15@gmail.com
}

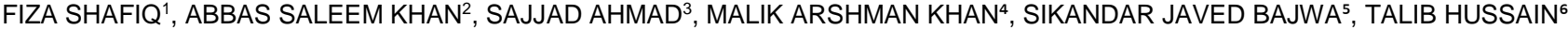

\begin{abstract}
Background: Oral cancer represents a significant component of the global cancer burden. It develops as a result of multiple risk factors. The main histological form of oral cancer is oral squamous cell carcinoma (OSCC). The disintegration of the extracellular matrix $(E C M)$ is one of the key hallmarks of cancer progression, which stimulates the invasion of tumoral cells into the neighbouring tissue. Matrix metalloproteinases-9 (MMP-9) can degrade different elements of the ECM and basement membrane. Methods: A total of 35 histologically diagnosed cases of OSCC $(n=25)$ and healthy individuals $(n=10)$ as control were included. Immunohistochemical staining was performed on all cases. The results were analysed using SPSS version 20.

Results: Increased expression of MMP-9 was observed in OSCC cases ( $n=23 / 25 ; 92 \%)$ as compared to healthy individuals. Statistically, a significant relation was observed while comparing the grade of staining intensity and MMP-9 expression among OSCC cases $(p=0.001)$.

Conclusion: The present study is planned to assess the immunohistochemical expression of MMP-9 in diagnosed cases of OSCC and healthy individuals. Increased expression of MMP-9 was observed in OSCC as compared to healthy individuals. Evaluation of MMP-9 could be of clinical importance in OSCC and can be used as a diagnostic predictive marker of OSCC. Keywords: Oral squamous cell carcinoma; Matrix Metalloproteinase -9; Immunohistochemistry
\end{abstract}

\section{INTRODUCTION}

Oral cancer is the sixth leading cancer worldwide ${ }^{1}$. The incidence rate of oral cancer varies geographically ${ }^{2}$. The mortality and incidence rate of oral cancer is higher in men than in women ${ }^{3}$. Most oral cancer cases occur between the ages of 50 and 75 years ${ }^{4-6}$. The commonest epithelial malignancy that occurs in the oral cavity is OSCC $(90 \%)^{7}$. In Pakistan, it is ranked third among the male and female population respectively. Amongst adults $(>18$ years), it is the second commonest malignancy ${ }^{8,9}$. The main histological form of oral cancer is oral squamous cell carcinoma (OSCC), which accounts for nearly $90 \%$ of all oral cancer cases ${ }^{9}$. The reported male to female ratio in Pakistan is $2: 1$. The average age of Pakistan's population, both males and females, is approximately $45-50$ years ${ }^{10}$. The common site of OSCC in Pakistan's population is the tongue, followed by buccal mucosa ${ }^{2}$.

The cancer results from abnormal changes in epithelial cells or an imbalance in the tumor microenvironment. Different types of cells, like endothelial cells, activated fibroblast, immune cells, basement membrane, and extracellular matrix, constitute the tumor microenvironment (TME). The remodeling of ECM helps the adjacent tissue invasion in the tumor microenvironment by cancer cells, making it an important aspect in the progression and behavior of cancer cells ${ }^{11,12}$. The five-year survival rate of OSCC is poor and is about $50 \%$ worldwide. The survival rate is low in patients with advanced stage of oral cancer, even after treatment like radiotherapy or chemoradiotherapy. It may be a diagnosis of OSCC in the late stage, so there should be an authentic marker that could predict the disease in the early stage ${ }^{2}$.

Matrix Metalloproteinases (MMPs) are one of the important enzymes which are both zinc and calcium-dependent endopeptidases. These are the factors that regulate cell behavior and enhance the cancer development process through modulation of angiogenesis, proliferation, invasion, and migration ${ }^{13}$.

They are required for remodeling of tissue and disintegration of ECM, which includes type IV collagen, the main component of the basement membrane ${ }^{13}$. MMPs, especially MMP-9, belong to the gelatinase group, also introduced as $92 \mathrm{KDa}$ type IV collagenase/gelatinase, promote tumor growth by forming new blood vessels. They disturb the morphology of tissue that allows

Received on 11-05-2021

Accepted on 27-09-2021 the growth of a tumor and they degrade the basement membrane. This enables metastasis and overexpression of MMP-9 in OSCC has prognostic value ${ }^{13,14}$. MMP-9 disintegrates type IV collagen and promotes HNSCC invasion. MMP-9 has been presented as an invasion and infiltration pattern marker of OSCC at the invasive front. MMP-9 can cleave multiple proteins such as TGF $\beta$, Ecadherin, chemokines, and cell surface receptors ${ }^{15}$.

The aim of this study is to assess the immunohistochemical expression (staining intensity and percentage of positive cells) of MMP-9 in a tissue sample of diagnosed cases of oral squamous cell carcinoma and normal oral mucosa.

Inclusion criteria

Group A: Histopathologically diagnosed cases of oral squamous cell carcinoma were graded as well-differentiated squamous cell carcinoma, moderately differentiated squamous cell carcinoma, and poorly differentiated squamous cell carcinoma.

Group B: Individuals with histologically diagnosed normal mucosa. The mucosa was acquired after getting informed consent from healthy individuals visiting the mentioned centers for already planned minor surgical procedures in which extra normal oral epithelial tissue is excised and discarded as part of treatment plan. Exclusion Criteria

Group A: Cases of OSCC of the oropharynx, hypopharynx, nasopharynx, and intraoral minor salivary gland, oral mucosal melanoma, and jaw bone malignancies (other than OSCC).

Group B: Subjects that were unwilling to participate in the study.

\section{METHODOLOGY}

The present study was performed in the oral pathology department of PMC after permission from IRB. The cases were collected from the archives of the PMC histopathology lab and PIMS histopathology lab. The study includes patients of all age groups with histopathologically diagnosed OSCC cases. Immunohistochemistry was used for the study to evaluate the expression of the IHC marker MMP-9 in OSCC cases. A total of 25 histologically diagnosed cases of OSCC $(n=25)$ and 10 cases of healthy individuals were included in the study. Slides were prepared for MMP-9 monoclonal antibody by immunohistochemically staining. Antigen retrieval was done by inserting in citrate buffer. Immunoreactivity of MMP-9 was assessed by marking a case as positive or negative on the basis of staining intensity and percentage of positive cells. All stained 
sections were evaluated using $4 x, 10 x$ and $40 x$ objectives of a light microscope to demonstrate positivity for MMP-9 expression. Data was analyzed and IHC scoring was performed. Mean, median, and standard deviation for MMP-9 were calculated for the OSCC age group. Data was further examined for statistical significance using SPSS version 20.

\section{RESULT}

A total of 35 cases were studied using SPSS for analysis. Among them, 10 cases were from healthy individuals and 25 cases were from OSCC. The mean age of presentation for cases of OSCC was 56.52 (SD \pm 12.24). Single sample "t" test revealed that the difference in the ages of cases of healthy individuals was found to be statistically significant $(<0.05)$ and insignificant for cases of OSCC (Table 1). Most of the cases of OSCC $(n=20)$ were presented at an age above 50 years. The M: $\mathrm{F}$ observed for the cases of OSCC was $(10: 15=1.6: 1)$, and healthy control $(6: 4=1.5: 1)$ respectively. A statistically significant $(p=0.04)$ relation was observed while comparing the age of study participants (Table 2). Among cases of OSCC, only 2 cases (8\%) did not express MMP-9 expression while the remaining 23 cases $(92 \%)$ expressed MMP-9 staining. Statistically significant relation $(p=0.001)$ was observed while comparing the grades of staining intensity and MMP-9 expressions (Table 3).

Statistically, an insignificant relation was observed between the MMP-9 tissue immunoreactivity and the site of OSCC lesion $(p=0.675)$, WHO grading system $(p=0.485)$, ITF grading system $(p=0.426)$, and LPI $(p=0.145)$ (Table 4). Among the cases of OSCC, all the cases $(n=10 ; 40 \%)$ that developed from the buccal mucosa, vestibule of the mouth, and retromolar area expressed MMP-9 expression in a tissue sample. Among cases of OSCC, all the lesions diagnosed as MDSCC ( $n=7 ; 28 \%)$, PDSCC expressed MMP-9. Regarding lymphoplasmacytic infiltration; OSCC cases which are marked MMP-9 positive (23/25;92\%) immunohistochemically, $8(32 \%)$ cases expressed many large patches, followed by continuous rim formation in $8(32 \%)$ cases and few patches in $7(28 \%)$ cases. Among cases of OSCC, 23 cases showed immunoexpression of MMP-9 in tissue samples, while normal oral mucosa did not show expression of MMP-9 in their tissue samples (Table 5)
Figure 1 (a-d) A. Photomicrograph of H\&E staining of OSCC (Magnification X10) B. Photomicrograph of IHC staining of OSCC show increased expression of MMP-9 immunoreactivity (Magnification X10) C. Photomicrograph of IHC staining of normal oral mucosa shows no expression of MMP-9 (Magnification X40) D. Photomicrograph of H\&E staining of the normal oral mucosa (Magnification X10).

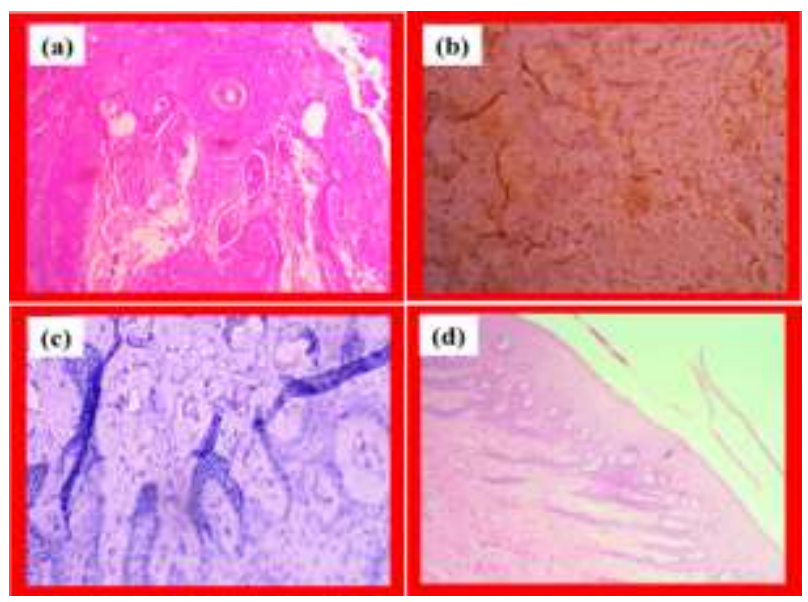

Table 1: Mean and SD of Age of Study Population

\begin{tabular}{|l|l|l|l|l|l|}
\hline Group & Minimum & Maximum & Mean & SD & p-value \\
\hline A & 23 & 82 & 56.52 & 12.24 & $\mathrm{P}=0.200$ \\
\hline B & 32 & 73 & 49.9 & 13.46 & $\mathrm{P}=0.021$ \\
\hline
\end{tabular}

Table 2: Description (Age, Gender) of the Study Population
\begin{tabular}{|l|l|l|l|}
\hline $\begin{array}{l}\text { Study } \\
\text { variable }\end{array}$ & $\begin{array}{l}\text { Group } \\
\text { (OSCC)(n=25) }\end{array}$ & $\begin{array}{l}\text { Group B (Healthy } \\
\text { individuals)(n=10) }\end{array}$ & $\begin{array}{l}\text { P value (Chi } \\
\text { square test) }\end{array}$ \\
\hline Age & $2(8 \%)$ & $3(30 \%)$ & \multirow{2}{*}{0.04} \\
\hline $20-40$ & $3(12 \%)$ & $3(30 \%)$ & \\
\hline $41-50$ & $10(40 \%)$ & $2(20 \%)$ & \\
\hline $51-60$ & $10(40 \%)$ & $2(20 \%)$ & 0.55 \\
\hline$>60$ & $10(40 \%)$ & $6(60 \%)$ & \\
\cline { 1 - 2 } Gender & $15(60 \%)$ & $4(40 \%)$ & \\
\hline Male & Female &
\end{tabular}

Table 3: MMP-9 Expression and Staining Intensity in OSCC

\begin{tabular}{|l|l|l|l|l|l|}
\hline \multirow{2}{*}{$\begin{array}{l}\text { Grades of Staining } \\
\text { Intensity }\end{array}$} & Absence of staining or no expression & Grade 1= >25 \% & $\begin{array}{l}\text { Grade 2 } \\
\mathbf{2 3 - 5 0 \%}\end{array}$ & $\begin{array}{l}\text { Grade 3 } \\
\mathbf{2 5 0} \%\end{array}$ & \multicolumn{1}{|c|}{ Total } \\
\hline No staining & $2(8 \%)$ & - & - & - & $2(8 \%)$ \\
\hline Weak staining & - & $4(16 \%)$ & $1(4 \%)$ & - & $5(20 \%)$ \\
\hline Moderate staining & - & $3(12 \%)$ & $1(4 \%)$ & $1(4 \%)$ & $5(20 \%)$ \\
\hline Intense staining & - & $4(16 \%)$ & $5(20 \%)$ & $4(16 \%)$ & $13(52 \%)$ \\
\hline Total & $2(8 \%)$ & $11(44 \%)$ & $7(28 \%)$ & $5(20 \%)$ & $25(100 \%)$ \\
\hline
\end{tabular}

$\mathrm{P}$ value 0.001

Table 4: Clinico-Pathological Features of OSCC among cases of Group A

\begin{tabular}{|c|c|c|c|c|c|c|}
\hline \multicolumn{3}{|c|}{ Clinicopathological Features } & \multicolumn{2}{|c|}{$\begin{array}{c}\text { MMP-9 } \\
\text { immunoreactivity }\end{array}$} & \multicolumn{2}{|c|}{ Statistics } \\
\hline ICD-10 Code & \multicolumn{2}{|l|}{ Site of development of OSCC lesions } & Negative & \begin{tabular}{l|l} 
& Positive
\end{tabular} & Total & $P$ value \\
\hline $\mathrm{COO}$ & \multicolumn{2}{|l|}{ Malignant neoplasm of Lip (External and inner aspects of lip, commissure of lip) } & - & $1(4 \%)$ & $1(4 \%)$ & \multirow{6}{*}{0.675} \\
\hline C01 & \multicolumn{2}{|l|}{ Base of tongue (Posterior third, dorsal surface of base of tongue) } & $1(4 \%)$ & $3(12 \%)$ & $4(16 \%)$ & \\
\hline $\mathrm{C} 02$ & $\begin{array}{l}\text { Specified parts of tongue (Borders, anterior-two-thirds, dorsal and ventral } \\
\text { surfaces of tongue) }\end{array}$ & & $1(4 \%)$ & $6(24 \%)$ & $7(28 \%)$ & \\
\hline $\mathrm{C} 03$ & \multicolumn{2}{|l|}{ Gum (Upper and lower gum) } & - & $2(8 \%)$ & $2(8 \%)$ & \\
\hline C04 & \multicolumn{2}{|l|}{ Floor of mouth (Anterior and lateral floor of mouth) } & - & $1(4 \%)$ & $1(4 \%)$ & \\
\hline $\mathrm{C} 06$ & \multicolumn{2}{|l|}{$\begin{array}{l}\text { Other and unspecified parts of mouth (Cheek mucosa, vestibule of mouth, } \\
\text { retromolar area) }\end{array}$} & - & $10(40 \%)$ & $10(40 \%)$ & \\
\hline \multicolumn{7}{|c|}{ WHO Grading System } \\
\hline \multicolumn{3}{|c|}{ WDSCC } & $2(8 \%)$ & $13(52 \%)$ & $15(66 \%)$ & \multirow{3}{*}{0.485} \\
\hline MDSCC & & & - & $7(28 \%)$ & $7(28 \%)$ & \\
\hline PDSCC & & & - & $3(12 \%)$ & $3(12 \%)$ & \\
\hline \multicolumn{7}{|c|}{ Invasive tumor front grading system } \\
\hline \multicolumn{2}{|c|}{ G1 } & - & & $3(12 \%)$ & $3(12 \%)$ & \multirow[t]{2}{*}{0.426} \\
\hline G2 & & $2(4 \%)$ & & $12(48 \%)$ & $14(56 \%)$ & \\
\hline
\end{tabular}




\begin{tabular}{|l|l|l|l|}
\hline G3 & - & \multicolumn{2}{|l|}{$8(32 \%)$} \\
\hline Grades of lymphoplasmacytic infiltrate & $-(32 \%)$ \\
\hline Marked(Continuous rim) & - & $8(32 \%)$ \\
\hline Moderate(Many large patches) & - & $8(32 \%)$ \\
\hline Slight(A few patches) & $2(8 \%)$ & $8(32 \%)$ \\
\hline None(No infiltration found) & - & $7(28 \%)$ & $8(32 \%)$ \\
\hline & $9(36 \%)$ & -145 \\
\hline
\end{tabular}

Table 5: MMP-9 Immunoreactivity in Tissue Samples of OSCC and Normal Oral Mucosa

\begin{tabular}{|l|l|l|}
\hline MMP-9 Immunoreactivity & OSCC & Normal Oral Mucosa \\
\hline Positive & $23(92 \%)$ & - \\
\hline Negative & $2(8 \%)$ & 10 \\
\hline Total & $25(100 \%)$ & 10 \\
\hline
\end{tabular}

\section{DISCUSSION}

Globally, oral cancer is one of the most common malignant tumors $^{16}$. The common histological variant of oral cancer is oral squamous cell carcinoma ${ }^{17}$. Mostly, the OSCC is preceded by an oral potentially malignant lesion ${ }^{19}$. Biomarkers can play an important role in the diagnosis of OSCC. MMP-9 is a key hallmark in oral cancer that can be used in the early prediction of the disease $^{17}$. In the present study, evaluation of MMP-9 in tissue samples was performed among cases of OSCC and healthy individuals to assess its predictive value in early diagnosis of OSCC.

The present study observed a mean age of 56.52 for OSCC cases and a male-female ratio of 1.6:1 was observed. This finding is supported by studies reported by Mirza et al., and Gul et al., who observed a male-female ratio of $1.6: 1^{6,18}$. While this finding is contrary to Beena et al., who observed that most cases were reported in the younger age group $(77 \%)^{19}$. This finding is contrary to Hosagadde et al who observed that the young age (21-30 years) group was most commonly affected and also observed that males were more affected than females $(2.5: 1)^{20}$..

Regarding the site distribution, the most commonly involved sites by OSCC were the buccal mucosa, vestibule of the mouth, and retromolar area (40\%) followed by the tongue. Our study showed results (Table 4 ) which are consistent with the studies reported by Majumdar et al., (30\%) and Siriwardena et al., who observed buccal mucosa $(50 \%)$ was commonly affected ${ }^{21,22}$. Beena et al. observed that the tongue (65\%) and gingiva (59\%) were more commonly affected ${ }^{23}$.

The most common histopathologically variant of OSCC reported by our study was WDSCC $(40 \%)$ followed by MDSCC (28\%) and PDSCC (12\%). Our study showed results (Table 4) which are in accordance with other studies reported, such as WDSCC (35\%) by Siriwardena et al and Gul et al (42.5\%) of WDSCC $^{18,22}$. These findings were different from some previous studies reported by Remin et al who showed increased cases among moderately (42\%) and poorly differentiated squamous cell carcinomas $(15 \%)^{24}$. Our study observed a statistically significant relationship while comparing the grades of staining intensity and MMP-9 expression with the development of oral squamous cell carcinoma (Table 3 ). This observation is consistent with other studies reported by Jose et $a^{25,26}$. Our study showed that MMP-9 was not expressed in normal mucosa among healthy individuals. The present study revealed results which are consistent with other studies reported by Gkouveris et $\mathrm{al}^{15}$. Our study results are inconsistent with another study that observed mild expression in normal mucosa ${ }^{27}$.

In this present study, we have compared MMP-9 expression between normal oral mucosa and OSCC cases. There was a statistically significant infiltration of MMP-9 in OSCC cases as compared to the normal oral mucosa group. The inconsistent results of our present study may be due to the small sample size, lower number of cases of PDSCC, methodology used, and inclusion and exclusion criteria among the cases.

\section{CONCLUSIONS}

MMP-9 is reported to be one of the key indicators in the development of oral cancer. The present study showed that there was increased expression of MMP-9 in OSCC cases as compared to the normal oral mucosa. Early diagnosis of OSCC and its premalignant forms are the keys to improving the survival rate of OSCC and preventing the risk of conversion of oral potentially malignant lesions into malignancy. The present study revealed that immunohistochemical evaluation of MMP-9 could be a prognostic marker in OSCC and could be used for future cancer bio therapeutics. These findings will improve the treatment protocol and prognosis of patients in the future.

Ethical Approval: Approval of study was taken from the board of advanced studies and research (BASR) at Riphah International University. The ethical assent for the study was given by the institutional review board (IRB) of Prime Foundation Pakistan. Written informed consent was also obtained from patients or attendants after explaining the aims, objectives, methods, and possible outcomes of the present study.

\section{REFERENCES}

1. Sasahira, T. and T. Kirita, Hallmarks of cancer-related newly prognostic factors of oral squamous cell carcinoma. International journal of molecular sciences, 2018. 19(8): p. 2413.

2. Sahaf, R., et al., A study of 89 cases of oral squamous cell carcinoma presenting at Teaching Hospitals of Lahore, Pakistan. JPDA, 2017. 26(01): p. 27

3. Bray, F., et al., Global cancer statistics 2018: GLOBOCAN estimates of incidence and mortality worldwide for 36 cancers in 185 countries. CA: a cancer journal for clinicians, 2018. 68(6): p. 394-424.

4. Diz, P., et al., Oral and pharyngeal cancer in Europe: Incidence, mortality and trends as presented to the Global Oral Cancer Forum. Translational Research in Oral Oncology, 2017. 2: p. $2057178 \times 17701517$.

5. Al Moustafa, A.-E., Development of oral cancer: risk factors and prevention strategies. 2017: Springer.

6. Dissanayake, U., Malignancy grading of invasive fronts of oral squamous cell carcinomas: correlation with overall survival. Translational Research in Oral Oncology, 2017. 2: p. $2057178 \times 17708874$.

7. Al-Jaber, A., L. Al-Nasser, and A. El-Metwally, Epidemiology of oral cancer in Arab countries. Saudi medical journal, 2016. 37(3): p. 249.

8. Idrees, R., et al., Cancer prevalence in Pakistan: meta-analysis of various published studies to determine variation in cancer figures resulting from marked population heterogeneity in different parts of the country. World journal of surgical oncology, 2018. 16(1): p. 1-11.

9. Boxberg, M., et al., Tumour budding activity and cell nest size determine patient outcome in oral squamous cell carcinoma: proposal for an adjusted grading system. Histopathology, 2017. 70(7): p. 11251137.

10. Riaz, F., et al., Risk factors of oral cancer in Lahore, Pakistan: A case control design. Proceeding SZPGMI Vol, 2015. 29(1): p. 47-54.

11. Walker, C., E. Mojares, and A. del Río Hernández, Role of extracellular matrix in development and cancer progression. International journal of molecular sciences, 2018. 19(10): p. 3028

12. Poltavets, V., et al., The role of the extracellular matrix and its molecular and cellular regulators in cancer cell plasticity. Frontiers in oncology, 2018. 8: p. 431.

13. Bizu, G. and B.M. Habte, Effect of medications-related beliefs on adherence to treatment of type /I diabetes mellitus in a primary healthcare setting, Addis Ababa, Ethiopia. International Journal of Pharmaceutical Sciences and Research, 2016. 7(1): p. 144.

14. Karabencheva-Christova, T.G., C.Z. Christov, and G.B. Fields, Collagenolytic Matrix Metalloproteinase Structure-Function Relationships: Insights From Molecular Dynamics Studies. Advances in protein chemistry and structural biology, 2017. 109: p. 1-24. 
15. Gkouveris, I., et al., Matrix metalloproteinases in head and neck cancer: current perspectives. Metalloproteinases In Medicine, 2017. 4: p. 47-61.

16. Nagai, H. and Y.H. Kim, Cancer prevention from the perspective of global cancer burden patterns. Journal of thoracic disease, 2017. 9(3): p. 448.

17. Richards, D., Malignant transformation rates in oral lichen planus. Evidence-based dentistry, 2018. 19(4): p. 122-122.

18. Gul, $\mathrm{H}$., et al., Epidemiology and pathological trends in oral squamous cell carcinoma in a local tertiary care hospital.Int J Community Med Public Health, 2017. 4(12): p. 4440-4444.

19. Beena, V., et al., Oral squamous cell carcinoma in patients younger than 40 years: $A 10$ year retrospective study. International Journal of Scientific Study, 2016. 4(4): p. 150-153.

20. Hosagadde, S., J. Dabholkar, and N. Virmani, A clinicopathological study of oral potentially malignant disorders. Journal of Head \& Neck Physicians And Surgeons, 2016. 4(1): p. 29.

21. Majumdar, B., et al., Clinico-pathological prognosticators in oral squamous cell carcinoma: An update. Translational Research in Oral Oncology, 2017. 2: p. 2057178X17738912.
22. Siriwardena, B., et al., An evaluation of clinical and histopathological aspects of patients with oral submucous fibrosis in the background of oral squamous cell carcinoma.BioMed research international, 2018. 2018.

23. Mello, F.W., et al., Intraoral potentially malignant disorders in a Brazilian oral pathology service: epidemiological, clinical, and histopathological findings. Journal of oncology, 2018. 2018.

24. Rehm, J. and S. Imtiaz, Alcohol consumption as a risk factor for global burden of disease. A narrative review.Subst Abuse Treat Prev Policy, 2016. 11(1): p. 37.

25. Jose, D. and D.R. Mane, Correlation of matrix metalloproteinase- 9 expression with morphometric analysis of mucosal vasculature in oral squamous cell carcinoma, oral epithelial dysplasia, and normal oral mucosa. International journal of health sciences, 2018. 12(6): p. 36.

26. Atla, B., et al., Matrix metalloproteinase-9 (MMP-9) as prospective histopathological and molecular biomarker for oral squamous cell carcinoma. International Journal of Research in Medical Sciences, 2018. 6(8): p. 2801.

27. Chandolia, B., S.K. Basu, and M. Kumar, Can MMP-9 be a prognosticator marker for oral squamous cell carcinoma? Journal of clinical and diagnostic research: JCDR, 2016. 10(1): p. ZC09. 